\title{
A Neural Approach for Modeling Continuous Time Sequences with Intermittent Observations
}

\author{
Vinayak Gupta \\ IIT Delhi, India \\ Srikanta Bedathur \\ IIT Delhi, India \\ Sourangshu Bhattacharya \\ IIT Kharagpur, India
}

Abir De
IIT Bombay, India

\begin{abstract}
A large fraction of data generated via human activities such as online purchases, health records, spatial mobility etc. can be represented as continuous-time event sequences (CTES) i.e. sequences of discrete events over a continuous time. Learning neural models over CTES is a non-trivial task as it involves modeling the ever-increasing event timestamps, inter-event time gaps, event types, and the influences between different events within and across different sequences. Moreover, existing sequence modeling techniques consider a complete observation scenario i.e. the event sequence being modeled is completely observed with no missing events - an ideal setting that is rarely applicable in real-world applications. In this paper, we highlight our approach[8] for modeling CTES with intermittent observations. Buoyed by the recent success of neural marked temporal point processes (MTPP) for modeling the generative distribution of CTES, we provide a novel unsupervised model and inference method for learning MTPP in presence of event sequences with missing events. Specifically, we first model the generative processes of observed events and missing events using two MTPP, where the missing events are represented as latent random variables. Then, we devise an unsupervised training method that jointly learns both the MTPP using variational inference. Experiments across real-world datasets show that our modeling framework outperforms state-of-the-art techniques for future event prediction and imputation. This work appeared in AISTATS 2021.
\end{abstract}

2012 ACM Subject Classification Information systems $\rightarrow$ Data stream mining

Keywords and phrases Time Series, Missing Data, Temporal Point Processes

Related Version http://proceedings.mlr.press/v130/gupta21a.html

\section{Motivation and Related Work}

Learning the dynamics of CTES is a non-trivial task with the neural models as it requires perpetual modeling of continuous-time and inter-event relationships $[4,9,18]$. Recent developments to marked temporal point processes (MTPP) $[2,14]$ have shown an outstanding potential to characterize asynchronous events localized in continuous time that appear in a wide range of applications in healthcare [13], spatial networks [4, 7, 10], web and social networks [3, 5, 6, 9], finance [1] and many more. However, modern MTPP models are limited to settings where the training data is completely observed i.e. no missing observations at all. While working with fully observed data is an ideal setting, this is not possible in many practical scenarios as the data collection procedure may miss events due to crawling restrictions by social media platforms, privacy restrictions, and budgetary factors. Thus, it results in the poor predictive performance of MTPP models $[4,18,19]$ that skirt this issue.

Statistical analysis in presence of missing data has been widely researched in literature in various contexts [11]. A recent line of methods $[12,16]$ focus on imputing missing data 

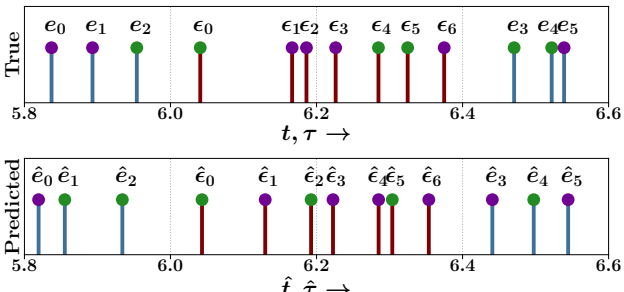

Figure 1 Qualitative Analysis of mark and time prediction performance of IMTPP. We show the true and events predicted by IMTPP for observed (bars in blue) and hidden events for the same sequence. Marks are represented using different colored (cyan and purple) circles.

in between a-priori available observed events, rather than predicting observed events in the face of missing events. Moreover, they deploy expensive learning and sampling mechanisms, which make them often intractable in practice.

\section{Intermittently-observed Marked Temporal Point Process}

In [8], we present an unsupervised model and inference method for learning neural MTPP over CTES with missing events. Specifically, we design a coupled-MTPP approach that first models the generative processes of both - observed events and missing events - where the missing events are represented as latent-random variables. Later, we jointly learn the distribution of all events via MTPP using variational inference.

The proposed model IMTPP(Intermittently-observed Marked Temporal Point Processes) models the generative distribution of observed events $\left(e_{k}\right)$ and missing events $\left(\epsilon_{r}\right)$ using MTPP denoted as $p(\bullet)$ and $q(\bullet)$ respectively. For generating future observed events, we follow a standard sampling procedure [15], however, the generation process of missing events is conditioned on the history as well as the next observed event. Specifically, we sample missing events between two observed events, till we reach the future observed event i.e. the MTPP are $-p\left(e_{k}\right)$ and $q\left(\epsilon_{r} \mid e_{k+1}\right)$ respectively. We learn the parameter by maximizing a variational lower bound or evidence lower bound (ELBO) of the log-likelihood.

$$
\left.\mathcal{L}=\mathbb{E}_{q} \sum_{k=0}^{K-1} \log p\left(e_{k+1}\right)-\sum_{k=0}^{K-1} K L\left[q\left(\epsilon_{r} \mid e_{k+1}\right)|| p_{r}\left(\epsilon_{r}\right)\right)\right],
$$

where $p_{r}, K L$ denote prior MTPP and KL-divergence respectively.

\section{Results and Future Work}

To highlight the ability of IMTPP to accurately identify the marks and times of missing events in a sequence, we use our trained model to predict both observed and missing events in Figure 1. We note that the predicted inter-arrival times and marks closely match with the true inter-arrival times. In our paper, we evaluated IMTPP across six datasets from different domains ranging Amazon, Twitter, Foursquare, NYC Taxi, and Stack-Overflow, and our observations were:

- IMTPP outperformed the state-of-the-art approaches [4, 18, 19], by up to $8 \%$ across all datasets for the mark and time prediction.

- The gains IMTPP had over other baselines we consistent even for forecasting.

- A scalability analysis showed that other CTES models took up to 24 hours training times, whereas the times for IMTPP were under 5 hours.

As future work, we aim to enhance IMTPP for event imputation and devise better ways to estimate the total number of missing events between each observed event. Moreover, we aim to incorporate self-attention [17] to better model the long-term dependencies between events. 


\section{References}

1 Emmanuel Bacry, Iacopo Mastromatteo, and Jean-François Muzy. Hawkes processes in finance. Market Microstructure and Liquidity, 2015.

2 Daryl J Daley and David Vere-Jones. An introduction to the theory of point processes: volume II: general theory and structure. Springer Science \& Business Media, 2007.

3 Abir De, Isabel Valera, Niloy Ganguly, Sourangshu Bhattacharya, and Manuel GomezRodriguez. Learning and forecasting opinion dynamics in social networks. In NeurIPS, 2016.

4 Nan Du, Hanjun Dai, Rakshit Trivedi, Utkarsh Upadhyay, Manuel Gomez-Rodriguez, and Le Song. Recurrent marked temporal point processes: Embedding event history to vector. In $K D D, 2016$.

5 Nan Du, Mehrdad Farajtabar, Amr Ahmed, Alexander J Smola, and Le Song. Dirichlet-hawkes processes with applications to clustering continuous-time document streams. In KDD, 2015.

6 Mehrdad Farajtabar, Jiachen Yang, Xiaojing Ye, Huan Xu, Rakshit Trivedi, Elias Khalil, Shuang Li, Le Song, and Hongyuan Zha. Fake news mitigation via point process based intervention. In $I C M L, 2017$.

7 Vinayak Gupta and Srikanta Bedathur. Region invariant normalizing flows for mobility transfer. In CIKM, 2021.

8 Vinayak Gupta, Srikanta Bedathur, Sourangshu Bhattacharya, and Abir De. Learning temporal point processes with intermittent observations. In AISTATS, 2021.

9 Srijan Kumar, Xikun Zhang, and Jure Leskovec. Predicting dynamic embedding trajectory in temporal interaction networks. In KDD, 2019.

10 Ankita Likhyani, Vinayak Gupta, PK Srijith, P Deepak, and Srikanta Bedathur. Modeling implicit communities from geo-tagged event traces using spatio-temporal point processes. In WISE, 2020.

11 Roderick JA Little and Donald B Rubin. Statistical analysis with missing data. John Wiley \& Sons, 2019.

12 Hongyuan Mei, Guanghui Qin, and Jason Eisner. Imputing missing events in continuous-time event streams. In ICML, 2019.

13 Marian-Andrei Rizoiu, Swapnil Mishra, Quyu Kong, Mark Carman, and Lexing Xie. Sirhawkes: on the relationship between epidemic models and hawkes point processes. In $W W W$, 2018.

14 Marian-Andrei Rizoiu, Lexing Xie, Scott Sanner, Manuel Cebrian, Honglin Yu, and Pascal Van Hentenryck. Expecting to be hip: Hawkes intensity processes for social media popularity. In $W W W, 2017$.

15 Oleksandr Shchur, Marin Biloš, and Stephan Günnemann. Intensity-free learning of temporal point processes. In ICLR, 2020.

16 Christian R. Shelton, Zhen Qin, and Chandini Shetty. Hawkes process inference with missing data. In $A A A I, 2018$.

17 Ashish Vaswani, Noam Shazeer, Niki Parmar, Jakob Uszkoreit, Llion Jones, Aidan N Gomez, Ł ukasz Kaiser, and Illia Polosukhin. Attention is all you need. In NeurIPS, 2017.

18 Qiang Zhang, Aldo Lipani, Omer Kirnap, and Emine Yilmaz. Self-attentive hawkes processes. In $I C M L, 2020$.

19 Simiao Zuo, Haoming Jiang, Zichong Li, Tuo Zhao, and Hongyuan Zha. Transformer hawkes process. In $I C M L, 2020$. 